time between inverse recall trials, as the Ss were not told whether they were right or wrong.

Each experimental group's mean recall times and the difference between each group's mean forward recall time (RB?) and its mean inverse recall time (?BC, $\mathrm{R}$ ? $\mathrm{C}$, or ??C) are presented in Table 2.

Because of the large differences in groups' variances, the Welch approximation (Winer, 1962 , p. 37) was used for a $t$ test. The average forward recall time was $1.88 \mathrm{sec}$, and the average inverse recall time was 2.89 sec. The probability of this occurring by chance is less than .01 (Welch $t=3.65$, df $=74$, two-tailed test).

To test our hypothesis about the effect of type of inverse question upon inverse recall time, we compared recall times for the three different inverse questions. Using the difference between inverse and forward times as the dependent variable for this analysis, we found that the mean recall time for the question ?BS-that is, for supplying the relation or attribute-was significantly less $(p<.05)$ than the mean recall times for the questions ?? $\mathrm{C}$ and $\mathrm{R}$ ?C.

This effect was present in all three experiments. In each case, the recall time for supplying the relation (attribute) was substantially less than the recall time for supplying the B-name (object) or B-name and relation. It is true that the effect, as measured by the difference between forward and inverse times, appears to be less pronounced in Experiment 3. However, as Table 2 reveals, this difference between experiments is not indicative of changes in inverse recall times, but is more the result of longer forward recall times in Experiment 3. The mean forward recall time for Experiment 3 was $2.18 \mathrm{sec}$, while for Experiment 2, it was 1.72 sec.

\section{DISCUSSION}

The differences in recall times among the inverse questions suggests a partial symmetry in accord with our hypothesis. It appears that Ss find it only slightly more difficult to supply a missing "attribute" (relation) in an AOV triad than to supply a missing "value" (C-name). On the other hand, Ss have much more difficulty in supplying a missing "object" (B-name). In information-processing terms, most Ss appear to have an operation called "find attribute" that is symmetrical to the previously proposed "find value" operation. Similarly, the significantly longer recall times for the inverse questions, $R$ ?C and ??C, indicates that Ss do not have a "find object" operation. To answer ??C explicitly would require both a "find attribute" and a "find object" operation.

Figure 1 contains a diagram of a typical S's behavior under this model. A leastsquares estimation of the times for the model's basic operations yielded $\mathrm{Tg}=0.12 \mathrm{Tv}, \mathrm{Ta}=1.38 \mathrm{Tv}$, and $\mathrm{Tc}=0.0 \mathrm{Tv}$. Hence, the predicted mean inverse-recall times are $\mathrm{T}_{\text {?BC }}=2.18, \mathrm{~T}_{\mathrm{R} \text { ?C }}=3.25$, and $\mathrm{T}_{\text {??C }}=3.17$

Could these results be explained as artifacts of the leaming procedure? Cumulative increases in the familiarity of C-words during learning clearly contributes to the speed of forward recall but would not explain the difference in inverse recall. The difference in inverse times was equally strong under Data Sets 1 and 2, indicating that differences in familiarity between the names and relations were not important. Experiment 2 ruled out the possibility that differences in meaningfulness between the relations and the names could account for the main effect, while Experiment 3 was used to rule out the possibility that (1) the order elements in the triad or (2) interference effects between B-names and $C$-names could account for the main effect.

\section{CONCLUSION}

The data support the contention that Ss have explicit operations for retrieving the value or attribute of a triad, but recall the object by generating feasible objects and testing them in the triad. One specific version of such a model is diagramed in Fig. 1. It is proposed that this model has developed in humans as a result of the differences in the restrictiveness of triadic recall questions and the differences in the frequency of occurrence of the questions in normal language usage.

\section{REFERENCE}

WINER, B. J. Statistical principles in experimental design. New York: McGraw-Hill, 1962. NOTES

1. This study was supported in part by Public Health Service Grants MH-07722 and MH-30,606-01A1(PS). The au thor is indebted to Herbert A. Simon for his advice in conducting these investigations and in preparing the manuscript. Miss Anita H. Retoff assisted in collection of the data.

2. Of course, there are many relations without this property, e.g., son, daughter.

\title{
Effect on backward masking of spatial separation between target and mask contours and of target size ${ }^{1}$
}

SUE I. COX, WILLIAM N. DEMBER, and MICHAEL F. SHERRICK, University of Cincinnati, Cincinnati, Ohio 45221

Werner's disk-ring patterns were used in a study of backward masking as a function of spatial separation between target and mask contours and of the absolute size of the target disk. This study differed from others that have investigated spatial separation in that a forced-choice indicator response was used. The results showed that (a) extent of masking increased with decreasing disk-ring separation; (b) masking was unrelated to disk size; and (c) disk size and disk-ring separation interacted to affect extent of masking. A possible interpretation of the interaction effect was proposed. The increase in masking with decreasing separation was discussed in terms of a lateral inhibition model of backward masking.

Backward masking refers to the decreased detectability of a target stimulus when it is followed closely in time by a subsequent stimulus. A frequently used configuration in masking studies employs a disk as the target stimulus and a ring fitting snugly around the disk as the mask.
Werner (1935) reported a failure to obtain masking when the diameter of the disk was less than the inner diameter of the ring (with the stimuli arranged concentrically). Kolers (1960), however, found that masking did occur with the disk and ring contours separated and that masking decreased as the separation increased. That relation has been confirmed by Wake (1967) and Streicher \& Pollack (1967), using slightly different procedures from Kolers and from each other. In all the above studies a yes-no indicator response was used to assess extent of masking.

It was the main purpose of the present experiment to investigate the effects of spatial separation of disk and ring on backward masking using a forced-choice indicator response, the advantages of which have been previously documented (Heckenmueller \& Dember, 1965). A second purpose was to examine possible effects on masking of the size of the stimuli and of the interaction between size and target-mask separation.

\section{SUBJECTS}

The paid Ss were four male and four female undergraduates at the University of 
Cincinnati, enrolled in the experimental psychology course. All had normal or corrected-to-normal vision. The Ss were trained on the task by being given a total of 1,000 trials on 2 days prior to the start of the actual experiment.

\section{STIMULI}

A set of stimuli, consisting of five disks and five rings, was prepared from black ink drawings on white index cards. The diameters of the disks as well as the inner diameter of the rings measured $6.0,8.5$, $11.0,13.5$, and $16.0 \mathrm{~mm}$, and at viewing distance of $1,180 \mathrm{~mm}$, subtended approximately $17,25,32,39$, and $47 \mathrm{~min}$ of a visual arc. From the five disks and five rings, 15 conditions were generated comprising disk-ring combinations in which the inner diameter of the ring was greater than or equal to the diameter of the disk. A given mask actually consisted of a pair of identical rings, arranged horizontally, to make possible the spatial forced-choice indicator response. The width of the wall of all rings was constant at $4 \mathrm{~mm}(12 \mathrm{~min})$. The distance between the centers of all pairs of rings was also constant, measuring $29 \mathrm{~mm}$ (1 deg $25 \mathrm{~min}$ ). Therefore, the distance between the outer edges of the ring pairs at their closest points ranged from $15 \mathrm{~mm}$ for the smallest pair of rings to $5 \mathrm{~mm}$ for the largest. The distances with which the study was most concerned-the distances between the inner edge of the ring and the disk-were $0 \mathrm{~mm}$ for five of the conditions, $1.25 \mathrm{~mm}$ ( $4 \mathrm{~min}$ ) for four of the conditions, $2.50 \mathrm{~mm}$ $(7 \mathrm{~min})$ for three of the conditions, $3.75 \mathrm{~mm}(11 \mathrm{~min})$ for two of the conditions, and $5.00 \mathrm{~mm}(15 \mathrm{~min})$ for one condition.

\section{APPARATUS AND PROCEDURE}

The stimuli were presented tachistiscopically (Scientific Prototype Model GB). The target stimuli were attached to a plate that could be moved horizontally. The apparatus included adjustable stops so that the positions at which the disk was centered in each of the two rings could be marked and the disk could easily be shifted between these two positions. Viewing was monocular.

The stimuli were presented in the order: fixation field, disk, ring, fixation field, with a target duration of $15 \mathrm{msec}$ and a mask duration of $100 \mathrm{msec}$; the termination of the target field defined the onset of the mask field (that is, the interstimulus interval was $0 \mathrm{msec}$ ). Approximately $5 \mathrm{sec}$ elapsed between presentations of the stimulus sequence and about $1 \mathrm{~min}$ between conditions. The fixation field was illuminated continuously except when the target or mask was present. Target, mask, and fixation fields were set at $25 \mathrm{ft}-\mathrm{L}$.

Each condition was viewed for 25 trials on each of 4 days for a total of 100 trials per

Table 1

Mean Per Cent Correct Disk Detection (Corrected for Guessing)

\begin{tabular}{lrrrrr}
\hline & \multicolumn{5}{c}{$\begin{array}{c}\text { Disk Diameter } \\
\text { (in mm) }\end{array}$} \\
$\begin{array}{lrrrrr}\text { Disk-Ring } \\
\begin{array}{l}\text { Separation } \\
\text { (in mm) }\end{array}\end{array}$ & 6.0 & 8.5 & 11.0 & 13.5 & 16.0 \\
\hline 0.00 & -11.5 & -2.3 & -5.0 & .5 & -4.3 \\
1.25 & 66.7 & 67.2 & 54.7 & 55.7 & \\
2.50 & 92.5 & 81.0 & 78.0 & & \\
3.75 & 97.2 & 97.7 & & & \\
5.00 & 97.0 & & & & \\
\hline
\end{tabular}

$S$ per condition. A trial consisted of the E's positioning the disk in one of the two rings, signalling the $S$ to push a button which initiated the stimulus sequence, and the S's saying "right" or "left" to indicate in which of the two rings the disk appeared, guessing if necessary. The location of the disk on a given trial was random as was the order of presentation of the conditions for a given $S$ on a given day. On each of the experimental days, each $S$ received several practice trials before the collection of the experimenta data.

\section{RESULTS}

The mean per cent correct detection, corrected for chance guessing, are presented in Table 1. The negative means indicate that the per cent correct detection was less than chance.

Since not all disks were used at each level of amount of separation, the tabled results contain empty cells. An analysis of variance procedure appropriate for the entire set of data was not contrived; instead, a two-way (complete) repeated-measures design was performed on the portion of the data from the intersection of the three smallest disk diameters and the three smallest target-mask separations. The F obtained for disk size was $1.841(\mathrm{df}=2 / 14, \mathrm{p}>.05)$; for distance, $F=64.49(\mathrm{df}=2 / 14, \mathrm{p}<.001)$; for the interaction between disk size and distance, $F=3.506(d f=4 / 28, p<.025)$.

From inspection of the means, it is evident that the detectability of the $6.0-\mathrm{mm}$ disk increases, relative to that of the $8.5 \mathrm{-mm}$ disk, with increasing target-mask separation; conversely, detectability of the $8.5-\mathrm{mm}$ disk decreases, relative to that of the $6.0 \mathrm{~mm}$ disk, as separation increases.

\section{DISCUSSION}

As expected, amount of masking does decrease with increase in separation between the disk and ring contours. However, in this study, as well as in others (Kolers, 1960; Wake, 1967; Streicher \& Pollack, 1967), some masking has been found to occur even at small target-mask separations, contrary to Werner's report of no masking when separation was greater than zero. This difference may reflect the greater sensitivity of the present method of measuring masking compared with phenomenal report as used by Werner.
The results also demonstrated that increasing the separation between disk and ring differentially affects the detectability of the 6.0 and the $8.5-\mathrm{mm}$ disks. In an attempt to account for this interaction, we computed the area separating disk and ring for the linear separations of $1.25,2.50$, and $3.75 \mathrm{~mm}$. The ratios of these areas for the $6.0 \mathrm{~mm}$ disk to the areas for the $8.0 \mathrm{-mm}$ disk are $.745, .773$, and .795 , respectively. Note that as linear separation increases by a constant amount, the area of separation increases at a greater rate for the $6.0 \mathrm{~mm}$ than for the $8.5-\mathrm{mm}$ disk. Thus, the interaction obtained between disk size and linear separation may simply be an artifact of the choice of separation measure and the nonlinear relation between distance and area. Unfortunately, distance and area cannot be varied independently of one another.

The main effect of target-mask separation is subject to more than one interpretation. We find the results interesting in that they. are consistent with an interpretation of the role of the mask based on a lateral inhibition model developed by Purcell, Stewart, \& Dember (1968). In essence, that interpretation conceives of the mask as protecting, or insulating the target from inhibitory effects imposed by the brightly illuminated area in the masking field that surrounds the area of the disk. Protecting the disk from that source of inhibition allows the disk area more fully to undergo "brightness reversal" and hence to be maximally subject to masking. Separating the target disk from its protecting mask decreases the extent of brightness reversal and therefore also decreases amount of masking.

\section{REFERENCES}

HECKENMUEL LER, E G. \& DEMBER, W.N. A forced choice indicator for use with Werner's disc-ring pattern in studies of backward masking. Psychonomic Science, 1965, 3, 167-168.

KOLERS, P. A. On visual masking (metacontrast): Dichoptic observation. American Joumal of Psychology, 1960, 63, 2-21.

PURCELL, D. G., STEWART, A. L., \& DEMBER, W.N. Spatial effectiveness of the mask: Lateral inhibition in visual backward masking. Perception \& Psychophysics, 1968, 4, 344-346. STREICHER, H. W., \& POLLACK, R. H. Backward figural masking as a function of intercontour distance. Psychonomic Science, 1967, 7, 69-70.

WAKE, T. The effect of separation and overlap between the disk and the ring upon the contour effect. Japanese Psychological Research, 1967, 9, 35-41.

WERNER, H. Studies on contour: I. Qualitative analysis. American Joumal of Psychology, $1935,47,40-64$.

\section{NOTE}

1. This research was supported in part by Grant NB 07622-03 from the National Institute of Neurological Diseases and Blindness. 